\title{
İş Tatmini Faktörlerini Belirlemeye Ve Analiz Etmeye Yönelik Olarak Çalışanların Çevrimiçi Değerlendirmelerinin Sınıflandırıcı Topluluklarına Dayalı Analizi
}

\author{
Ali Özdemir ${ }^{1}$, Aytuğ Onan ${ }^{2}$, Vildan Çınarlı Ergene ${ }^{3 *}$ \\ ${ }^{1}$ Matematik /Fen Edebiyat Fakültesi, Manisa Celal Bayar Üniversitesi, Manisa, Türkiye,(ORCID: 0000 -0001-9330-7084), acaozdemir@gmail.com \\ 2 Bilgisayar Mühendisliği / Mühendislik Fakültesi, İzmir Katip Çelebi Üniversitesi, İzmir, Türkiye, (ORCID: 0000-0002-9434-5880), aytugonan@gmail.com \\ 3* Matematik / Fen Bilimleri Enstitüsü, Manisa Celal Bayar Üniversitesi, Manisa, Türkiye (ORCID: 0000-0002-1220-3337), vildan.cinarli@gmail.com
}

(1st International Conference on Applied Engineering and Natural Sciences ICAENS 2021, November 1-3, 2021)

(DOI: 10.31590/ejosat.1008691)

ATIF/REFERENCE: Özdemir, A., Onan, A., \& Çınarlı Ergene, V. (2021). İş Tatmini Faktörlerini Belirlemeye Ve Analiz Etmeye Yönelik Olarak Çalışanların Çevrimiçi Değerlendirmelerinin Sınıflandıııcı Topluluklarına Dayalı Analizi. Avrupa Bilim ve Teknoloji Dergisi, (28), 531-538.

Öz

Bu çalışma kapsamında iş tatmini faktörlerini belirlemeye ve analiz etmeye yönelik olarak çalışanların çevrimiçi değerlendirmelerinin sınıflandırıcı topluluklarına dayalı analizi yapılışșır. Metin sınıflandırma işlemi, önceden tanımlanmış kategoriler veya sınıflar altında metin belgelerinin otomatik olarak sınıflandırılmasıdır. Bu kapsamda makine öğrenmesi teknikleri, dokümanlara anahtar kelimeler atamak ve bunları belirli kategorilerde sınıflandırmak için kullanılır. Makine öğrenmesi, belgeleri otomatik olarak kategorilere ayırmamızı sağlar.

Metin madenciliği, metinleri organize etmek amacıyla önceden tanımlanmış kategorilere dahil etme işlemidir. Son yıllarda dijital ortamdaki verinin artması, bu verilerden bilgi çıkarımını ve bilginin kullanılabilirliğinin artırılması üzerine olan çalışmaları hızlandırmıştır. Metinlerin verimli kullanılabilmesi ve aranılan bilgiye hızlıca ulaşılabilmesi için metinlerin kategorize edilmesi önem kazanmıştır. Büyük belge koleksiyonlarını elle düzenlemek son derece zaman alıcıdır ve hataya açıktır. Otomatik metin sınıflandırma uygulamaları bu noktada hızlı ve maliyeti düşük çözümler sunar. Metin sınıflandırma işleminin, istenmeyen mesaj veya elektronik postaları filtreleme, bilimsel makalelerin otomatik indekslenmesi, web sayfaların etiketlenmesi, belge türünün tanımlanması gibi uygulama alanları mevcuttur.

Çalışanların çevrimiçi değerlendirmelerinin sınıflandıııcı topluluklarına dayalı analizi yapılması amacıyla çalışan kişilerin çalışmış oldukları firmalar hakkındaki düşünceleri tr.indeed.com'da yayınlanan toplam 18 farklı firmadan elde edilen 3023 adet çevrimiçi değerlendirme elde edilmiştir. Her bir veri seti için, öznitelik temsili yöntemlerinden terim sıklığı (TF), terim varlığı (TP) ve TF-IDF ölçütleri için 1-gram, 2-gram ve 3-gram temsilleri ile toplam dokuz farklı veri temsili elde edilmiştir. Yapılan deneysel çalışmalarda beş temel sınıflandırma algoritması topluluk öğrenme yöntemleri ile birleştirilerek doğru sınıflandırma performansları incelenmiştir.

Anahtar Kelimeler: Sınıflandırıcı topluluğu, Metin madenciliği, Makine öğrenmesi

\section{Analysis of Employees' Online Assessments Based on Classifier Ensembles to Identify and Analyze Job Satisfaction Factors}

\begin{abstract}
Within the scope of this study, an analysis of the online evaluations of employees based on classifier communities was made in order to identify and analyze job satisfaction factors. Text classification is the automatic classification of text documents under predefined categories or classes. In this context, machine learning techniques are used to assign keywords to documents and classify them into specific categories. Machine learning allows us to automatically categorize documents.

Text mining is the process of including texts into predefined categories for the purpose of organizing them. The increase in data in the digital environment in recent years has accelerated the studies on extracting information from these data and increasing the usability of information. It has become important to categorize the texts in order to use the texts efficiently and to reach the sought information quickly. Manually editing large document collections is extremely time-consuming and error-prone. Automatic text classification applications offer fast and cost-effective solutions at this point. Text classification has application areas such as filtering spam or emails, automatic indexing of scientific articles, tagging web pages, and defining document type.
\end{abstract}

\footnotetext{
*Sorumlu Yazar:vildan.cinarli@gmail.com
} 
In order to analyze the online evaluations of the employees based on their classifier communities, 3023 online evaluations obtained from a total of 18 different companies, which were published on tr.indeed.com, were obtained. For each data set, a total of nine different data representations were obtained with 1-gram, 2-gram and 3-gram representations for term frequency (TF), term presence (TP) and TF-IDF criteria from the feature representation methods. In the experimental studies, the correct classification performances were examined by combining five basic classification algorithms with ensemble learning methods.

Keywords: Classifier ensemble, Text mining, Machine learning

\section{Giriş}

İșletmelerin hedeflerine ulașması ve kar elde etmesi öncelikle çalışanlar sayesinde olur. Yani işini seven, işini iyi yapan, yaptığı işten gurur duyan, işletmenin hedeflerini benimsemiş ve amaçlarını işletme amaçlarıyla bütünleştirmiş çalışanlara sahip işletmeler başarılı olacaklardır. $\mathrm{Bu}$ da çalışanların işinden duydukları tatmin derecesiyle ilişkilidir. İş tatmini yöneticiler için çalışanların işlerine karşı tutumlarının performans ve verimlilik üzerindeki etkisi açısından önemlidir. Çünkü iş tatminsizliğinin doğrudan neden olduğu davranışlar; işten ayrılma, devamsızlık, performans düşüklüğü, ruhsal ve fiziksel sağlikta bozulma şeklinde görülmektedir [1].

Günümüzde modern yönetim anlayışı çalışanın iş tatmini üzerinde önemle durmaktadır. Tüm kurumlarda kurum başarısı çalışanın iş doyumu ile doğru orantılıdır. Kurumların kullandıkları kaynaklar içinde en karmaşık ve değişken olanı insan gücüdür. Örgütsel davranış alanında iş tatmini, en çok önem verilen ve sıklıkla araştırılan bir davranış olarak görülmektedir $[2]$.

Yapay zekâ çözümleri her sektörde artarak devam etmektedir. Metin madenciliği son yıllarda hızla gelişen bir alan haline geldi ve bu alandaki uygulamalar yapay zekâ alanındaki uygulamaların büyük bir bölümünü oluşturmaktadır. Diğer fonksiyonel departmanlar gibi, insan kaynakları departmanlarının işletme yöneticileri çeşitli konularda kararlar almalıdır. Metinsel veriler de dahil olmak üzere daha fazla veri oluşturulup biriktikçe şirketleri ile ilgili karar verirken metin madenciliği kullanılarak bu tür verilerden uygulanabilir bilgi elde edilir [3].

İnsan kaynakları yönetiminde yapay zekâ teknolojilerinin kullanılması; iş için doğru adayları seçmekte ve yetenek kazanmalarına yardımcı olmakta, işyerinde çalışanların elde tutulma oranının tahmin edilmesine ve şirketteki idari personel üzerindeki yükü azaltmaya yardımcı olmaktadır. Aynı zamanda, hata olasılığını minimum seviyeye indirmeye, çalışanların daha yüksek performans ile katılım sağlamasına ve karar vermede önyargı davranışını en aza indirmeye yardımcı olmaktadır [4].

\section{Materyal ve Metot}

Sınıflandırma işlemi daha önce görülmemiş ve kategorisi bilinmeyen her bir örneğin, eğitim verisi kategorilerinden en uygun olan kategoriye atanması işlemidir [5]. Makine öğrenmesi açısından düşünüldüğünde ise, yapılan son araştırmalar, metin madenciliği gibi yöntemler üzerine olmuştur. Makine öğrenmesine ihtiyaç duyulmasının nedeni, elle kategorizasyonun pahalı ve zaman tüketen bir iş oluşudur ki, ayrıca elle sınıflandırmada, sınıflandırmayı yapan uzmanların vermiş oldukları kararlara bağlı olarak sonuçlar da değişmektedir [6].

Çalışanların şirketleri hakkında yaptıkları yorumlardan 1 ve 2 puan olanlar negatif, 3,4 ve 5 puanlar pozitif olarak değerlendirilmiş olup toplamda 18 farklı firmadaki 2321 pozitif yorum ve 702 negatif yorum tr.indeed.com'dan elde edilmiştir.
Elde edilen 3023 adet çevrimiçi değerlendirme ele alınarak sınıflandırıcı topluluklarına dayalı analizleri gerçekleştirilmiştir.

Öncelikle sistemde kullanılacak olan verilerin WEKA programında hataları düzeltilerek ön işlemden geçirilmiştir. Ön işlemden geçirilen çevrimiçi değerlendirmeler, öznitelik temsili yöntemlerinden terim sıklığ 1 (Term Frequency-TF), terim varlığ1 (Term Present-TP) ve TF-IDF ölçütleri için N-gram temsil modellerinden olan 1-gram, 2-gram ve 3-gram temsilleri ile toplam dokuz farklı veri temsili elde edilmiştir. Daha sonra çıkarılan öznitelikler ağırlıklandırılmıştır. Son olarak elde edilen veriler arff (Attribute Relational File Format) formatına dönüştürülerek sınıflandırma işlemi gerçekleştirilmiştir. Çevrimiçi değerlendirme yapılan firmaların adı ve kaç adet pozitif ve negatif yorum yapıldığını belirten bilgiler Tablo1'de verilmiştir:

Tablo 1. Çevrimiçi değerlendirme yapılan firmalara ait yorum sayllarl

\begin{tabular}{|l|l|l|l|}
\hline Firma adı & $\begin{array}{l}\text { Negatif } \\
\mathbf{( 1 - 2} \text { puan) }\end{array}$ & $\begin{array}{l}\text { Pozitif } \\
\mathbf{( 3 - 4 - 5} \text { puan })\end{array}$ & $\begin{array}{l}\text { Toplam } \\
\text { Firma } \\
\text { Sayıs }\end{array}$ \\
\hline VESTEL & 16 & 85 & 101 \\
\hline BOSCH & 3 & 37 & 40 \\
\hline ARÇELİK & 23 & 151 & 174 \\
\hline SIEMENS & 2 & 33 & 35 \\
\hline MC DONALDS & 54 & 151 & 205 \\
\hline BURGER KING & 49 & 170 & 219 \\
\hline DOMINOS & 21 & 13 & 89 \\
\hline KOTON & 43 & 0 & 173 \\
\hline LC WAIKIKI & 49 & 337 & 386 \\
\hline DE FACTO & 32 & 189 & 221 \\
\hline STARBUCKS & 8 & 86 & 94 \\
\hline $\begin{array}{l}\text { KAHVE } \\
\text { DÜNYASI }\end{array}$ & 16 & 38 & 54 \\
\hline TURKCELL & 24 & 0 & 158 \\
\hline $\begin{array}{l}\text { TURK } \\
\text { TELEKOM }\end{array}$ & 33 & 0 & 212 \\
\hline VODAFONE & 23 & 0 & 161 \\
\hline BIM A.Ş. & 39 & 113 & 152 \\
\hline A101 & 260 & 248 & 51 \\
\hline MIGROS & 7 & & \\
\hline
\end{tabular}

Çalışmamızda 5 temel sınıflandırıcı olarak NaiveBayesMultinomial, lazy.IBk, Random.forest, functions.Logistic ve functions.LibLINEAR algoritmaları kullanmış olup kullanılan öğrenme yöntemleri aşağıdaki gibidir; 
- $\mathrm{Bu} 5$ temel algoritmanın tümü Meta Adaboost algoritması için ayrı ayrı denenerek 9 konfigürasyon için 5 farklı analiz yapıldı.

- Benzer şekilde 5 temel algoritmanın tümü Meta Bagging algoritması için ayrı ayrı denenerek 9 konfigürasyon için 5 farklı analiz yapıldı.

- 5 temel algoritmanın tümü Meta Random Subspace algoritması için ayrı ayrı denenerek 9 konfigürasyon için 5 farklı analiz yapıldı.

- Stacking (L1 regularized L2 loss support vector classification) algoritması 5 algoritma kullanılarak Logistic ile çalıştırılarak 1 analiz yapıldı.

- Meta Vote algoritması ise içerisindeki 5 tane farklı kombinasyon (Liblinear+L1 regularized logistic regression) ile (meta.Vote AVG- meta.Vote PRODmeta.Vote MAJORITY- meta.Vote MIN- meta.Vote MAX ) çalıştırıldı.

Buna göre toplamda $21 * 9=189$ tane deney elde edilmiş oldu.

\section{Araştırma Sonuçları ve Tartışma}

Sınıflandırmaya ait deneysel sonuçlar Weka tabanlı Java uygulaması ile elde edilmiş olup beş temel sınıflandırma algoritması topluluk öğrenme yöntemleri ile birleştirilmiştir. $\mathrm{Bu}$ deneylerin sonuçlarına ait veriler tablolar ile gösterilmiştir (Tablo 2-6).

Tablo2.Temel algoritmaların AdaBoost sınıflandırıcı topluluğuna dayalı analizleri

\begin{tabular}{|c|c|c|c|c|c|}
\hline Algoritma & \multicolumn{5}{|c|}{ Veri seti için elde edilen sonuçlar } \\
\hline $\begin{array}{l}\text { meta.AdaBoostM1- } \\
\text { bayes.NaiveBayesMultinomial }\end{array}$ & Correctly Classified Instances & Precision & Recall & F-Measure & $\begin{array}{l}\text { Time taken to build } \\
\text { model: }\end{array}$ \\
\hline TF_bigram.arff & $75.1902 \%$ & 0,734 & 0,752 & 0,741 & 0.05 seconds \\
\hline TF_trigram.arff & $74.8594 \%$ & 0,707 & 0,749 & 0,717 & 0.03 seconds \\
\hline TF_unigram.arff & $75.2564 \%$ & 0,761 & 0,753 & 0,756 & 0.05 seconds \\
\hline TFIDF_bigram.arff & $71.8822 \%$ & 0,732 & 0,719 & 0,725 & 0.03 seconds \\
\hline TFIDF_trigram.arff & $71.8492 \%$ & 0,693 & 0,718 & 0,704 & 0.03 seconds \\
\hline TFIDF_unigram.arff & $74.0655 \%$ & 0,753 & 0,741 & 0,746 & 0.04 seconds \\
\hline TP_bigram.arff & $72.8746 \%$ & 0,732 & 0,729 & 0,73 & 0.04 seconds \\
\hline TP_trigram.arff & $74.6279 \%$ & 0,707 & 0,746 & 0,718 & 0.04 seconds \\
\hline TP_unigram.arff & $74.5617 \%$ & 0,754 & 0,746 & 0,749 & 0.04 seconds \\
\hline $\begin{array}{l}\text { meta.AdaBoostM1- } \\
\text { functions.LibLINEAR }\end{array}$ & Correctly Classified Instances & Precision & Recall & F-Measure & $\begin{array}{l}\text { Time taken to build } \\
\text { model: }\end{array}$ \\
\hline TF_bigram.arff & $74.3632 \%$ & 0,726 & 0,744 & 0,733 & 0.21 seconds \\
\hline TF_trigram.arff & $75.3887 \%$ & 0,701 & 0,754 & 0,708 & 0.14 seconds \\
\hline TF_unigram.arff & $73.5693 \%$ & 0,741 & 0,736 & 0,738 & 1.55 seconds \\
\hline TFIDF_bigram.arff & $73.4370 \%$ & 0,72 & 0,734 & 0,726 & 1.5 seconds \\
\hline TFIDF_trigram.arff & $74.5617 \%$ & 0,691 & 0,746 & 0,703 & 1.29 seconds \\
\hline TFIDF_unigram.arff & $73.4039 \%$ & 0,741 & 0,734 & 0,738 & 2.33 seconds \\
\hline TP_bigram.arff & $73.6024 \%$ & 0,722 & 0,736 & 0,728 & 0.31 seconds \\
\hline TP_trigram.arff & $74.9917 \%$ & 0,698 & 0,75 & 0,708 & 0.18 seconds \\
\hline TP_unigram.arff & $73.4039 \%$ & 0,741 & 0,734 & 0,737 & 2.25 seconds \\
\hline $\begin{array}{l}\text { meta.AdaBoostM1- } \\
\text { functions.Logistic }\end{array}$ & Correctly Classified Instances & Precision & Recall & F-Measure & $\begin{array}{l}\text { Time taken to build } \\
\text { model: }\end{array}$ \\
\hline TF_bigram.arff & $70.7906 \%$ & 0,705 & 0,708 & 0,706 & 1939.32 seconds \\
\hline TF_trigram.arff & $71.5184 \%$ & 0,681 & 0,715 & 0,695 & 267.4 seconds \\
\hline TF_unigram.arff & $67.4165 \%$ & 0,705 & 0,674 & 0,687 & 4692.38 seconds \\
\hline TFIDF_bigram.arff & $70.9229 \%$ & 0,706 & 0,709 & 0,708 & 1365.47 seconds \\
\hline TFIDF_trigram.arff & $71.5845 \%$ & 0,682 & 0,716 & 0,695 & 178.94 seconds \\
\hline TFIDF_unigram.arff & $67.4496 \%$ & 0,708 & 0,674 & 0,688 & 2137.57 seconds \\
\hline TP_bigram.arff & $70.7575 \%$ & 0,704 & 0,708 & 0,706 & 1223.26 seconds \\
\hline TP_trigram.arff & $71.3860 \%$ & 0,68 & 0,714 & 0,693 & 194.26 seconds \\
\hline
\end{tabular}




\begin{tabular}{|l|l|l|l|l|l|}
\hline TP_unigram.arff & $67.1849 \%$ & 0,706 & 0,672 & 0,686 & 2318.24 seconds \\
\hline $\begin{array}{l}\text { meta.AdaBoostM1- } \\
\text {.trees.RandomForest }\end{array}$ & Correctly Classified Instances & Precision & Recall & F-Measure & $\begin{array}{l}\text { Time taken to build } \\
\text { model: }\end{array}$ \\
\hline TF_bigram.arff & $75.5872 \%$ & 0,727 & 0,756 & 0,735 & 628.27 seconds \\
\hline TF_trigram.arff & $75.9510 \%$ & 0,706 & 0,76 & 0,709 & 953.79 seconds \\
\hline TF_unigram.arff & $81.2107 \%$ & 0,798 & 0,812 & 0,812 & 120.28 seconds \\
\hline TFIDF_bigram.arff & $75.8518 \%$ & 0,729 & 0,759 & 0,737 & 461.39 seconds \\
\hline TFIDF_trigram.arff & $76.0172 \%$ & 0,707 & 0,76 & 0,709 & 941.56 seconds \\
\hline TFIDF_unigram.arff & $80.6153 \%$ & 0,79 & 0,806 & 0,774 & 117.38 seconds \\
\hline TP_bigram.arff & $76.4472 \%$ & 0,736 & 0,764 & 0,743 & 453.26 seconds \\
\hline TP_trigram.arff & $75.9180 \%$ & 0,705 & 0,759 & 0,708 & 926.5 seconds \\
\hline TP_unigram.arff & $80.2845 \%$ & 0,785 & 0,803 & 0,77 & 161.31 seconds \\
\hline meta.AdaBoostM1-lazy Ibk & $\mathbf{C o r r e c t l y ~ C l a s s i f i e d ~ I n s t a n c e s ~}$ & $\mathbf{P r e c i s i o n}$ & Recall & F-Measure & $\begin{array}{l}\text { Time taken to build } \\
\text { model: }\end{array}$ \\
\hline TF_bigram.arff & $72.5438 \%$ & 0,69 & 0,725 & 0,703 & 12.22 seconds \\
\hline TF_trigram.arff & $75.4879 \%$ & 0,682 & 0,755 & 0,689 & 9.6 seconds \\
\hline TF_unigram.arff & $69.5336 \%$ & 0,669 & 0,695 & 0,68 & 27 seconds \\
\hline TFIDF_bigram.arff & $72.2461 \%$ & 0,687 & 0,722 & 0,7 & 10.28 seconds \\
\hline TFIDF_trigram.arff & $75.5210 \%$ & 0,683 & 0,755 & 0,69 & 9.26 seconds \\
\hline TFIDF_unigram.arff & $70.7244 \%$ & 0,666 & 0,707 & 0,682 & 29.05 seconds \\
\hline TP_bigram.arff & $74.9587 \%$ & 0,701 & 0,75 & 0,711 & 10.44 seconds \\
\hline TP_trigram.arff & $75.7195 \%$ & 0,686 & 0,757 & 0,69 & 9.21 seconds \\
\hline TP_unigram.arff & $70.7244 \%$ & 0,666 & 0,707 & 0,682 & 26.76 seconds \\
\hline
\end{tabular}

Tablo3.Stacking sinıflandırıcı topluluğuna dayalı analizler

\begin{tabular}{|l|l|l|l|l|l|}
\hline Algoritma & Correctly Classified Instances & Precision & Recall & F-Measure & $\begin{array}{l}\text { Time taken to } \\
\text { build model: }\end{array}$ \\
\hline meta.Stacking & & 0,786 & 0,803 & 0,768 & 1334.02 seconds \\
\hline TF_bigram.arff & $80.2845 \%$ & 0,77 & 0,783 & 0,714 & 2308.68 seconds \\
\hline TF_trigram.arff & $78.2666 \%$ & $\mathbf{0 , 8 2 6}$ & $\mathbf{0 , 8 3 7}$ & $\mathbf{0 , 8 2 3}$ & $\mathbf{5 4 1 . 9 6}$ seconds \\
\hline TF_unigram.arff & $\mathbf{8 3 . 6 5 8 6 \%}$ & 0,77 & 0,794 & 0,768 & 1343.01 seconds \\
\hline TFIDF_bigram.arff & $79.3913 \%$ & 0,724 & 0,77 & 0,693 & 2352.92 seconds \\
\hline TFIDF_trigram.arff & $77.0427 \%$ & 0,814 & 0,825 & 0,816 & 525.22 seconds \\
\hline TFIDF_unigram.arff & $82.5339 \%$ & 0,786 & 0,804 & 0,776 & 1329.51 seconds \\
\hline TP_bigram.arff & $80.4499 \%$ & 0,758 & 0,782 & 0,72 & 2327.57 seconds \\
\hline TP_trigram.arff & $78.2005 \%$ & 0,822 & 0,833 & 0,821 & 523.04 seconds \\
\hline TP_unigram.arff & $83.3278 \%$ & & &
\end{tabular}

Tablo4. Temel algoritmaların Bagging sinfflandırıcı topluluğuna dayalı analizleri

\begin{tabular}{|l|l|l|l|l|l|}
\hline \multicolumn{1}{|c|}{ Algoritma } & \multicolumn{5}{c|}{ Veri seti için elde edilen sonuçlar } \\
\hline $\begin{array}{l}\text { meta.Bagging- } \\
\text { bayes.NaiveBayesMultinomial }\end{array}$ & Correctly Classified Instances & Precision & Recall & F-Measure & $\begin{array}{l}\text { Time taken to build } \\
\text { model: }\end{array}$ \\
\hline TF_bigram.arff & $80.5491 \%$ & 0,788 & 0,805 & 0,774 & 0.1 seconds \\
\hline TF_trigram.arff & $78.2997 \%$ & 0,778 & 0,783 & 0,711 & 0.02 seconds \\
\hline TF_unigram.arff & $83.2947 \%$ & 0,822 & 0,833 & 0,823 & 0.02 seconds \\
\hline TFIDF_bigram.arff & $76.4803 \%$ & 0,76 & 0,765 & 0,762 & 0.02 seconds \\
\hline TFIDF_trigram.arff & $72.1469 \%$ & 0,697 & 0,721 & 0,707 & 0.01 seconds \\
\hline TFIDF_unigram.arff & $80.6153 \%$ & 0,806 & 0,806 & 0,806 & 0.03 seconds \\
\hline TP_bigram.arff & $80.0198 \%$ & 0,781 & 0,8 & 0,783 & 0.01 seconds \\
\hline
\end{tabular}




\begin{tabular}{|c|c|c|c|c|c|}
\hline TP_trigram.arff & $76.9765 \%$ & 0,727 & 0,77 & 0,724 & 0.02 seconds \\
\hline TP_unigram.arff & $82.4677 \%$ & 0,816 & 0,825 & 0,819 & 0.02 seconds \\
\hline $\begin{array}{l}\text { meta.Bagging- } \\
\text {.functions.LibLINEAR }\end{array}$ & Correctly Classified Instances & Precision & Recall & F-Measure & $\begin{array}{l}\text { Time taken to build } \\
\text { model: }\end{array}$ \\
\hline TF_bigram.arff & $76.5465 \%$ & 0,735 & 0,765 & 0,741 & 0.3 seconds \\
\hline TF_trigram.arff & $76.1164 \%$ & 0,701 & 0,761 & 0,701 & 0.14 seconds \\
\hline TF_unigram.arff & $78.7297 \%$ & 0,776 & 0,787 & 0,781 & 0.65 seconds \\
\hline TFIDF_bigram.arff & $73.0069 \%$ & 0,719 & 0,73 & 0,724 & 1.35 seconds \\
\hline TFIDF_trigram.arff & $74.6279 \%$ & 0,686 & 0,746 & 0,698 & 1.23 seconds \\
\hline TFIDF_unigram.arff & $74.1317 \%$ & 0,751 & 0,741 & 0,746 & 1.56 seconds \\
\hline TP_bigram.arff & $75.2564 \%$ & 0,726 & 0,753 & 0,735 & 0.22 seconds \\
\hline TP_trigram.arff & $75.5541 \%$ & 0,697 & 0,756 & 0,703 & 0.17 seconds \\
\hline TP_unigram.arff & $77.2742 \%$ & 0,768 & 0,773 & 0,77 & 0.82 seconds \\
\hline $\begin{array}{l}\text { meta.Bagging- } \\
\text { functions.Logistic }\end{array}$ & Correctly Classified Instance & Precision & Recall & F-Measure & $\begin{array}{l}\text { Time taken to build } \\
\text { model: }\end{array}$ \\
\hline TF_bigram.arff & $70.3275 \%$ & 0,711 & 0,703 & 0,707 & 472.89 seconds \\
\hline TF_trigram.arff & $74.0324 \%$ & 0,692 & 0,74 & 0,705 & 248.9 seconds \\
\hline TF_unigram.arff & $67.5157 \%$ & 0,705 & 0,675 & 0,688 & 208.56 seconds \\
\hline TFIDF_bigram.arff & $70.3936 \%$ & 0,711 & 0,704 & 0,707 & 475.89 seconds \\
\hline TFIDF_trigram.arff & $74.1317 \%$ & 0,693 & 0,741 & 0,706 & 246.83 seconds \\
\hline TFIDF_unigram.arff & $67.0857 \%$ & 0,699 & 0,671 & 0,683 & 208.5 seconds \\
\hline TP_bigram.arff & $70.0298 \%$ & 0,711 & 0,7 & 0,705 & 452.7 seconds \\
\hline TP_trigram.arff & $73.9332 \%$ & 0,692 & 0,739 & 0,705 & 238.57 seconds \\
\hline TP_unigram.arff & $66.9864 \%$ & 0,698 & 0,67 & 0,682 & 204.44 seconds \\
\hline meta.Bagging -lazy.IBk & Correctly Classified Instances & Precision & Recall & F-Measure & $\begin{array}{l}\text { Time taken to build } \\
\text { model: }\end{array}$ \\
\hline TF_bigram.arff & $73.2716 \%$ & 0,687 & 0,733 & 0,701 & 0.05 seconds \\
\hline TF_trigram.arff & $75.7195 \%$ & 0,686 & 0,757 & 0,69 & 0.01 seconds \\
\hline TF_unigram.arff & $70.6583 \%$ & 0,668 & 0,707 & 0,683 & 0.04 seconds \\
\hline TFIDF_bigram.arff & $72.8746 \%$ & 0,683 & 0,729 & 0,698 & 0.03 seconds \\
\hline TFIDF_trigram.arff & $75.7526 \%$ & 0,687 & 0,758 & 0,691 & 0.02 seconds \\
\hline TFIDF_unigram.arff & $71.5845 \%$ & 0,662 & 0,716 & 0,681 & 0.03 seconds \\
\hline TP_bigram.arff & $75.2564 \%$ & 0,697 & 0,753 & 0,705 & 0.04 seconds \\
\hline TP_trigram.arff & $75.7856 \%$ & 0,686 & 0,758 & 0,69 & 0.02 seconds \\
\hline TP_unigram.arff & $71.5845 \%$ & 0,662 & 0,716 & 0,681 & 0.03 seconds \\
\hline $\begin{array}{l}\text { meta.Bagging- } \\
\text { trees.RandomForest }\end{array}$ & Correctly Classified Instances & Precision & Recall & F-Measure & $\begin{array}{l}\text { Time taken to build } \\
\text { model: }\end{array}$ \\
\hline TF_bigram.arff & $77.3073 \%$ & 0,741 & 0,773 & 0,744 & 883.3 seconds \\
\hline TF_trigram.arff & $76.7450 \%$ & 0,715 & 0,767 & 0,704 & 1085.66 second \\
\hline TF_unigram.arff & $81.4092 \%$ & 0,811 & 0,814 & 0,777 & 353.69 seconds \\
\hline TFIDF_bigram.arff & $77.2081 \%$ & 0,739 & 0,772 & 0,743 & 914 seconds \\
\hline TFIDF_trigram.arff & $76.6126 \%$ & 0,711 & 0,766 & 0,702 & 1577.89 seconds \\
\hline TFIDF_unigram.arff & $81.5746 \%$ & 0,816 & 0,816 & 0,777 & 334.85 seconds \\
\hline $\mathrm{TP}$ _bigram.arff & $77.4727 \%$ & 0,743 & 0,775 & 0,746 & 899.55 seconds \\
\hline TP_trigram.arff & $76.7119 \%$ & 0,714 & 0,767 & 0,703 & 1575.37 seconds \\
\hline TP_unigram.arff & $81.5084 \%$ & 0,818 & 0,815 & 0,775 & 339.37 seconds \\
\hline
\end{tabular}


Tablo5. Temel algoritmaların RandomSubSpace sınıflandırıcı topluluğuna dayalı analizi

\begin{tabular}{|c|c|c|c|c|c|}
\hline Algoritma & \multicolumn{5}{|c|}{ Veri seti için elde edilen sonuçlar } \\
\hline $\begin{array}{l}\text { meta.RandomSubSpace- } \\
\text { functions.Logistic }\end{array}$ & Correctly Classified Instances & Precision & Recall & F-Measure & $\begin{array}{l}\text { Time taken to } \\
\text { build model: }\end{array}$ \\
\hline TF_bigram.arff & $77.7373 \%$ & 0,747 & 0,777 & 0,749 & 425.04 seconds \\
\hline TF trigram.arff & $75.5872 \%$ & 0,702 & 0,756 & 0,708 & 60.93 seconds \\
\hline TF_unigram.arff & $80.5491 \%$ & 0,787 & 0,805 & 0,787 & 153.86 seconds \\
\hline TFIDF_bigram.arff & $77.5719 \%$ & 0,744 & 0,776 & 0,746 & 426.64 seconds \\
\hline TFIDF trigram.arff & $75.1571 \%$ & 0,695 & 0,752 & 0,704 & 62.15 seconds \\
\hline TFIDF_unigram.arff & $80.8138 \%$ & 0,791 & 0,808 & 0,79 & 130.94 seconds \\
\hline TP bigram.arff & $77.6712 \%$ & 0,747 & 0,777 & 0,75 & 399.04 seconds \\
\hline TP_trigram.arff & $75.4548 \%$ & 0,699 & 0,755 & 0,706 & 54.91 seconds \\
\hline TP_unigram.arff & $80.0860 \%$ & 0,782 & 0,801 & 0,784 & 138.53 seconds \\
\hline $\begin{array}{l}\text { meta.RandomSubSpace- } \\
\text { functions.LibLINEAR }\end{array}$ & Correctly Classified Instances & Precision & Recall & F-Measure & $\begin{array}{l}\text { Time taken to } \\
\text { build model: }\end{array}$ \\
\hline TF_bigram.arff & $78.8621 \%$ & 0,762 & 0,789 & 0,751 & 0.36 seconds \\
\hline TF_trigram.arff & $76.2818 \%$ & 0,7 & 0,763 & 0,695 & 0.3 seconds \\
\hline TF_unigram.arff & $81.4423 \%$ & 0,799 & 0,814 & 0,794 & 0.78 seconds \\
\hline TFIDF_bigram.arff & $77.4727 \%$ & 0,744 & 0,775 & 0,747 & 1.47 seconds \\
\hline TFIDF_trigram.arff & $76.1826 \%$ & 0,706 & 0,762 & 0,705 & 1.11 seconds \\
\hline TFIDF_unigram.arff & $80.4499 \%$ & 0,788 & 0,804 & 0,791 & 2.1 seconds \\
\hline TP_bigram.arff & $78.2997 \%$ & 0,753 & 0,783 & 0,75 & 0.44 seconds \\
\hline TP_trigram.arff & $76.2818 \%$ & 0,707 & 0,763 & 0,704 & 0.35 seconds \\
\hline TP_unigram.arff & $81.1446 \%$ & 0,795 & 0,811 & 0,795 & 0.94 seconds \\
\hline $\begin{array}{l}\text { meta.RandomSubSpace- } \\
\text { RandomForest }\end{array}$ & Correctly Classified Instances & Precision & Recall & F-Measure & $\begin{array}{l}\text { Time taken to } \\
\text { build model: }\end{array}$ \\
\hline TF_bigram.arff & $78.6636 \%$ & 0,758 & 0,787 & 0,749 & 531.26 seconds \\
\hline TF_trigram.arff & $76.9765 \%$ & 0,72 & 0,77 & 0,697 & 1388 seconds \\
\hline TF_unigram.arff & $81.5415 \%$ & 0,817 & 0,815 & 0,776 & 249.26 seconds \\
\hline TFIDF_bigram.arff & $78.6636 \%$ & 0,758 & 0,787 & 0,746 & 544.84 seconds \\
\hline TFIDF_trigram.arff & $77.2412 \%$ & 0,73 & 0,772 & 0,701 & 1400.58 seconds \\
\hline TFIDF_unigram.arff & $81.6077 \%$ & 0,821 & 0,816 & 0,776 & 231.78 seconds \\
\hline TP_bigram.arff & $78.5643 \%$ & 0,757 & 0,786 & 0,748 & 591.22 seconds \\
\hline TP_trigram.arff & $76.9765 \%$ & 0,72 & 0,77 & 0,698 & 756.34 seconds \\
\hline TP_unigram.arff & $81.8062 \%$ & 0,825 & 0,818 & 0,778 & 379.82 seconds \\
\hline $\begin{array}{l}\text { meta.RandomSubSpace- } \\
\text { NaiveBayesMultinomial }\end{array}$ & Correctly Classified Instances & Precision & Recall & F-Measure & $\begin{array}{l}\text { Time taken to } \\
\text { build model: }\end{array}$ \\
\hline TF_bigram.arff & $79.4244 \%$ & 0,785 & 0,794 & 0,741 & 0.27 seconds \\
\hline TF_trigram.arff & $77.4065 \%$ & 0,798 & 0,774 & 0,683 & 0.69 seconds \\
\hline TF_unigram.arff & $82.8316 \%$ & 0,819 & 0,828 & 0,807 & 0.66 seconds \\
\hline TFIDF_bigram.arff & $78.3989 \%$ & 0,77 & 0,784 & 0,775 & 0.41 seconds \\
\hline TFIDF_trigram.arff & $74.4955 \%$ & 0,706 & 0,745 & 0,717 & 0.32 seconds \\
\hline TFIDF_unigram.arff & $81.0453 \%$ & 0,804 & 0,81 & 0,807 & 0.6 seconds \\
\hline TP_bigram.arff & $80.2514 \%$ & 0,787 & 0,803 & 0,766 & 0.41 seconds \\
\hline TP_trigram.arff & $77.9027 \%$ & 0,773 & 0,779 & 0,701 & 0.32 seconds \\
\hline TP_unigram.arff & $82.7985 \%$ & 0,816 & 0,828 & 0,814 & 0.59 seconds \\
\hline $\begin{array}{l}\text { meta.RandomSubSpace- } \\
\text { LazyIBK }\end{array}$ & Correctly Classified Instances & Precision & Recall & F-Measure & $\begin{array}{l}\text { Time taken to } \\
\text { build model: }\end{array}$ \\
\hline TF_bigram.arff & $76.7450 \%$ & 0,72 & 0,767 & 0,716 & 0.27 seconds \\
\hline
\end{tabular}




\begin{tabular}{|l|l|l|l|l|l|}
\hline TF_trigram.arff & $76.9434 \%$ & 0,72 & 0,769 & 0,7 & 0.33 seconds \\
\hline TF_unigram.arff & $77.2742 \%$ & 0,73 & 0,773 & 0,712 & 0.58 seconds \\
\hline TFIDF_bigram.arff & $76.9765 \%$ & 0,725 & 0,77 & 0,718 & 0.42 seconds \\
\hline TFIDF_trigram.arff & $76.8773 \%$ & 0,717 & 0,769 & 0,699 & 0.35 seconds \\
\hline TFIDF_unigram.arff & $77.4727 \%$ & 0,763 & 0,775 & 0,69 & 0.59 seconds \\
\hline TP_bigram.arff & $77.3404 \%$ & 0,732 & 0,773 & 0,714 & 0.38 seconds \\
\hline TP_trigram.arff & $76.7780 \%$ & 0,714 & 0,768 & 0,699 & 0.34 seconds \\
\hline TP_unigram.arff & $77.5389 \%$ & 0,764 & 0,775 & 0,692 & 0.59 seconds \\
\hline
\end{tabular}

Tablo6.Vote sinıflandırıcı topluluğuna dayalı analizler

\begin{tabular}{|c|c|c|c|c|c|}
\hline Algoritma & \multicolumn{5}{|c|}{ Veri seti için elde edilen sonuçlar } \\
\hline meta.Vote AVG & Correctly Classified Instances & Precision & Recall & F-Measure & $\begin{array}{l}\text { Time taken to } \\
\text { build model: }\end{array}$ \\
\hline TF_bigram.arff & $78.7297 \%$ & 0,759 & 0,787 & 0,749 & 105.46 seconds \\
\hline TF_trigram.arff & $76.8111 \%$ & 0.716 & 0.768 & 0.7 & 86 seconds \\
\hline TF_unigram.arff & $81.7400 \%$ & 0,803 & 0,817 & 0,797 & 43.74 seconds \\
\hline TFIDF_bigram.arff & $76.6457 \%$ & 0.743 & 0.766 & 0.75 & 47.93 seconds \\
\hline TFIDF_trigram.arff & $74.9587 \%$ & 0,696 & 0,75 & 0,706 & 188.09 seconds \\
\hline TFIDF_unigram.arff & $79.5237 \%$ & 0.78 & 0.795 & 0.784 & 17.09 seconds \\
\hline TP_bigram.arff & $78.8621 \%$ & 0,761 & 0,789 & 0,754 & 93.01 seconds \\
\hline TP_trigram.arff & $76.5134 \%$ & 0.71 & 0.765 & 0.704 & 102 seconds \\
\hline TP_unigram.arff & $81.6408 \%$ & 0,801 & 0,816 & 0,798 & 38.57 seconds \\
\hline meta.Vote PROD & Correctly Classified Instances & Precision & Recall & F-Measure & $\begin{array}{l}\text { Time taken to } \\
\text { build model: }\end{array}$ \\
\hline TF_bigram.arff & $73.2054 \%$ & 0.789 & 0.806 & 0.755 & 45.31 seconds \\
\hline TF_trigram.arff & 73.6024 & 0.762 & 0.782 & 0.69 & 74.18 second \\
\hline TF_unigram.arff & $74.4625 \%$ & 0,823 & 0,836 & 0,817 & 57.11 seconds \\
\hline TFIDF_bigram.arff & $69.2689 \%$ & 0.742 & 0.77 & 0.75 & 44.56 seconds \\
\hline TFIDF_trigram.arff & $72.6100 \%$ & 0,689 & 0,752 & 0,701 & 296.1 seconds \\
\hline TFIDF_unigram.arff & $67.7804 \%$ & 0.772 & 0.785 & 0.777 & 17.5 seconds \\
\hline TP_bigram.arff & $71.3199 \%$ & 0,777 & 0,802 & 0,759 & 128.82 seconds \\
\hline TP_trigram.arff & $72.5438 \%$ & 0.763 & 0.784 & 0.695 & 75.2 seconds \\
\hline TP_unigram.arff & $72.6761 \%$ & 0.763 & 0.784 & 0.695 & 40.42 seconds \\
\hline meta.Vote MAJORITY & Correctly Classified Instances & Precision & Recall & F-Measure & $\begin{array}{l}\text { Time taken to } \\
\text { build model: }\end{array}$ \\
\hline TF_bigram.arff & $79.1598 \%$ & 0,767 & 0,792 & 0,756 & 72.41 seconds \\
\hline TF_trigram.arff & $77.0096 \%$ & 0,722 & 0,77 & 0,704 & 79.05 seconds \\
\hline TF_unigram.arff & $82.4347 \%$ & 0,813 & 0,824 & 0,802 & 44.61 seconds \\
\hline TFIDF_bigram.arff & $76.9434 \%$ & 0,744 & 0,769 & 0,75 & 75.48 seconds \\
\hline TFIDF_trigram.arff & $74.9587 \%$ & 0,689 & 0,75 & 0,699 & 124.51 seconds \\
\hline TFIDF_unigram.arff & $80.5491 \%$ & 0,788 & 0,805 & 0,788 & 27.98 seconds \\
\hline TP_bigram.arff & $78.7297 \%$ & 0,76 & 0,787 & 0,755 & 74.97 seconds \\
\hline TP_trigram.arff & $76.4142 \%$ & 0,711 & 0,764 & 0,708 & 132.91 seconds \\
\hline TP_unigram.arff & $82.0046 \%$ & 0,807 & 0,82 & 0,798 & 29.42 seconds \\
\hline meta.Vote MIN & Correctly Classified Instances & Precision & Recall & F-Measure & $\begin{array}{l}\text { Time taken to } \\
\text { build model: }\end{array}$ \\
\hline
\end{tabular}


Tablo 6'nın devamı

\begin{tabular}{|l|l|l|l|l|l|}
\hline TF_bigram.arff & $73.2385 \%$ & 0,789 & 0,806 & 0,755 & 135.3 seconds \\
\hline TF_trigram.arff & $73.6355 \%$ & 0,763 & 0,782 & 0,69 & 230.42 seconds \\
\hline TF_unigram.arff & $74.4625 \%$ & 0,823 & 0,836 & 0,817 & 52.34 seconds \\
\hline TFIDF_bigram.arff & $69.3351 \%$ & 0,742 & 0,77 & 0,749 & 129.83 seconds \\
\hline TFIDF_trigram.arff & $72.6100 \%$ & 0,689 & 0,752 & 0,701 & 227.61 seconds \\
\hline TFIDF_unigram.arff & $67.8465 \%$ & 0,772 & 0,784 & 0,777 & 49.94 seconds \\
\hline TP_bigram.arff & $71.3199 \%$ & 0,777 & 0,802 & 0,759 & 71.51 seconds \\
\hline TP_trigram.arff & $72.5438 \%$ & 0,763 & 0,784 & 0,695 & 129.84 seconds \\
\hline TP_unigram.arff & $72.6761 \%$ & 0,809 & 0,824 & 0,807 & 29.3 seconds \\
\hline meta.Vote MAX & Correctly Classified Instances & Precision & Recall & F-Measure & $\begin{array}{l}\text { Time } \\
\text { build model: }\end{array}$ \\
\hline TF_bigram.arff & & & & & to \\
\hline TF_trigram.arff & 76.8111 & 0,739 & 0,768 & 0,745 & 180.19 seconds \\
\hline TF_unigram.arff & $76.1495 \%$ & 0,705 & 0,761 & 0,705 & 30.1 seconds \\
\hline TFIDF_bigram.arff & $78.3328 \%$ & 0,778 & 0,783 & 0,781 & 105.75 seconds \\
\hline TFIDF_trigram.arff & $72.3123 \%$ & 0,721 & 0,723 & 0,722 & 161.77 seconds \\
\hline TFIDF_unigram.arff & $73.8339 \%$ & 0,686 & 0,738 & 0,7 & 32.15 seconds \\
\hline TP_bigram.arff & $72.5438 \%$ & 0,753 & 0,725 & 0,736 & 103.44 seconds \\
\hline TP_trigram.arff & $75.1571 \%$ & 0,73 & 0,752 & 0,737 & 124 seconds \\
\hline TP_unigram.arff & $75.5210 \%$ & 0,7 & 0,755 & 0,707 & 29.55 seconds \\
\hline
\end{tabular}

Makine öğrenmesi teknikleri ile hızlı ve güvenilir sekilde tahminlemeler yapılabilmektedir. Makine öğrenmesine ait birçok algoritma mevcut olup, problemin kaynağına ve veri sayısına göre hangi algoritmanın kullanılacağına karar verilmektedir. Farklı algoritmalar kullandığımız verisetine göre özgüllük, duyarlılık ve F-ölçütü değerleri üretmektedir. Kullandığımız verisetine göre bu değerler göz önünde bulundurularak farklı algoritmalar arasından en iyi uyum sağlayan algoritma tercih edilerek sonuç iyileştirilir ve zaman maliyeti azaltılır.

\section{Sonuç}

İş tatmini faktörlerini belirlemeye ve analiz etmeye yönelik olarak çalışanların çevrimiçi değerlendirmelerinin makine öğrenmesine dayalı analizini elde etmek amacıyla gerçekleştirilen çalışmalar hakkında bilgi vermektedir. Çalışanların şirketleri hakkında yaptıkları yorumlardan 1 ve 2 puan olanlar negatif, 3,4 ve 5 puanlar pozitif olarak değerlendirilmiş olup toplamda 18 firmadaki 2321 pozitif yorum ve 702 negatif yorum ele alınarak analizleri gerçekleştirilmiştir.

Metin belgesindeki özniteliklerin belirlenmesinde terim varlığı, terim sıklığı gibi temel temsil yöntemleri ve 1-gram, 2gram, 3-gram modelleri dikkate alınarak incelenen veri seti için farklı temsil yöntemlerinin doğru sınıflandırma başarımları, Fölçütü, duyarlılık, hassasiyet ölçütleri 5 temel sınıflandırıcı aracılığıyla karşılaştırmalı olarak değerlendirilmiştir.

Yapılan bu analizler sonucunda en yüksek başarı oranı Tablo 3'de görüleceği üzere $83.6586 \%$ başarı oranı ile meta.Stacking algoritmasında $\mathrm{TF}$ _unigram.arff komfigürasyonu ile elde edilmiş olup deney sonucunun alınması 541.96 saniye sürmüştür. $\mathrm{Bu}$ deneyden elde edilen duyarlılık değeri 0,826 ; hassasiyet değeri 0,837 ve F-ölçütü değer, 0,823 olup Terim sıklığı (Term
Frequency) konfigürasyonu ve 1-gram modeli ile en yüksek başarımı vermiştir. Analizler sonucunda en düşük başarı oranı ise Tablo 4'te görülen $66.9864 \%$ başarı oranı ile meta.Bagging functions.Logistic algoritmasından elde edilerek deneyin yapılması 204.44 saniye sürmüştür. Analiz sonucu elde edilen bu en düşük başarı oranı TP_unigram.arff konfigürasyonu ile yani terim varlığ 1 (Term Present) ve 1-gram modeli kullanılarak elde edilmiş olup sırasıyla duyarlılık;hassasiyet ve F-ölçütü değerleri 0,$698 ; 0 ; 67$ ve 0,682 olarak bulunmuştur.

\section{Kaynakça}

[1] Akşit Aşık, Nuran (2010), "Çalışanların İş Doyumunu Etkileyen Bireysel Ve Örgütsel Faktörler İle Sonuçlarına İlişkin Kavramsal Değerlendirme", Türk İdare Dergisi, 467(Haziran), 31-51.

[2] Luthans, F., Baack, D. ve Taylor, L. (1992). Organizational Commitment: Analysis of Antecedents, Human Relations.

[3] S. Strohmeier, F. Piazza, Domain driven data mining in human resource management: a review of currentresearch, Expert Systems with Applications 40 (7) (2013) 2410-2420.

[4] Yawalkar, V. V., (2019), "Study of Artificial Intelligence and its role in Human Resource Management", International Journal of Research and Analytical Reviews (IJRAR), pp.2024).

[5] Sebastiani, Fabrizio. "Machine learning in automated text categorization." ACM computing surveys (CSUR) 34.1 (2002): 1-47.

[6] U. Özkaya, L. Seyfi. (2021), "Yere Nüfuz Eden Radar B Tarama Görüntülerinin Az Parametreye Sahip Konvolüsyonel Sinir Ağı İle Değerlendirilmesi” Geomatik, 6(2), 84-92. 